\title{
Low field magnetic relaxation of superconducting $\mathrm{C}_{60} \mathrm{Rb}_{3}$
}

\author{
L.M. Wang ${ }^{a}$, H.C. Yang ${ }^{a}$, J.H. Hwang ${ }^{b}$, C.Y. Mou ${ }^{b}$, and H.E. Horng ${ }^{c}$ \\ aDepartment of Physics, National Taiwan University, Taipei, Taiwan,R.O.C.* \\ bepartment of Chemistry, National Taiwan University, Taipei, Taiwan,R.O.C. \\ CDepartment of Physics, National Taiwan Normal University, Taipei, Taiwan,R.O.C.*
}

The relaxation of magnetization $\mathrm{M}$ in superconducting $\mathrm{C}_{60} \mathrm{Rb}_{3}$ have been measured at different temperature ranges in low magnetic fields below $T_{C}$ to explore the flux pinning. The measurements were performed with a superconducting quantum interference magnetometer. The applied magnetic field was $300 \mathrm{G}-600 \mathrm{G}$ and the measured time at each temperature was $1 \times 10^{4} \mathrm{sec}$. M versus $\ln$ shows a linear behavior for $t \geq 3600 \mathrm{sec}$. The data were analyzed in the framework of the flux pinning model of Anderson-Kim. The analyses give the activation energy of $\mathrm{U}(5 \mathrm{~K}) \approx 20 \mathrm{mev}$, and the critical current density based on $\mathrm{J}_{\mathrm{C}}(\mathrm{T}) \propto[\mathrm{dM} / \mathrm{dlnt}] \times[\mathrm{U} / \mathrm{kT}]$ shows $\mathrm{J}_{\mathrm{c}} \propto(1-\mathrm{t})^{\mathrm{m}}$, with the exponent $\mathrm{m} \approx 2.0$. The results are discussed

\section{INTRODUCTION}

Recently, it has been discovered that dopings of $\mathrm{C}_{60}$ with the alkali metals form a new class of superconductor [1]. The transition temperature is $\mathrm{T}_{\mathrm{c}}$ $=18 \mathrm{~K}$ for $\mathrm{C}_{60} \mathrm{~K}_{3}[2]$ and $\mathrm{T}_{\mathrm{C}}=28 \mathrm{~K}$ for $\mathrm{C}_{60} \mathrm{Rb}_{3}[3]$. The first findings were suggestive of a type-\| superconductor, then the critical field, the field penetration depth $\lambda$ and the coherence length $\xi$ of these materials were measured [4,5]. In this paper we report the magnetic relaxation of $\mathrm{C}_{60} \mathrm{Rb}_{3}$, and derive the effective pinning energy $U$ and the critical current density Jc based on the flux creep model of Anderson-Kim

\section{EXPERIMENTAL PROCEDURE}

$\mathrm{C}_{60} \mathrm{Rb}_{3}$ samples were prepared in a way similar to that reported originally by Kratschmer et al. [6]. A SQUID magnetometer (Quantum Design Inc.) was employed to measure the magnetization $M$ as a function of time in an applied field, $\mathrm{H}=300 \mathrm{G}$ or $600 \mathrm{G}$. The sample was first zero-field-cooled to a desired temperature $\mathrm{T}$ below the transition temperature $T_{c}=27 \mathrm{~K}$; a magnetic field was then applied to the sample. The magnetization of the sample was measured as a function of time $t$.

\section{RESULTS AND DISCUSSION}

FIgure 1 shows the magnetic relaxation data as a funtion of $t$ at $\mathrm{T}=9,11$, and $13 \mathrm{~K}$ respectively in a magnetic field of $300 \mathrm{G}$. The common feature of these curves was that the magnetization data decrease as one increases $t$ and a linear behavior in $M(t)$ versus $\ln (t)$ for $t \geq 3600$ sec. The slope. $S(T)=$ $\mathrm{dM} / \mathrm{d} \ln$, varies considerably at different temperatures.

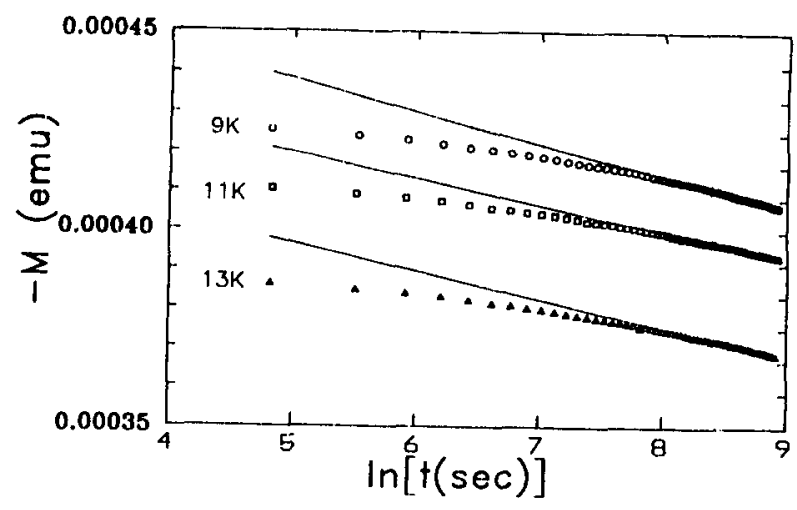

Figurel. Magnetization vs $\ln (t)$ at a given field $\mathrm{H}=300 \mathrm{G}$, and the temperatures are indicated.

Using $S(T)$, we calculated the flux-creep apparent activation energy based on the flux creep model of Anderson Kim [7] which can be written as

$$
\mathrm{U}=\mathrm{kTM}(0)[\mathrm{dM} / \mathrm{dln}(\mathrm{t})]^{-1},
$$

where $M(0)$ is the initial magnetization. Figure 2

\footnotetext{
*This work is supported by National Science Council of R.O.C. under Contract No. NSC 81-0212-MD03-516.
} 
shows the temperature dependence of $U(T)$ derived from $\mathrm{Eq}(1)$. One can see that as one decreases $T, U(T)$ increases with $T$ and reaches a $U_{\max }$ and then decreases with $T$. $U_{\max }(17 \mathrm{~K}) \approx 77.5 \mathrm{meV}$ in $300 \mathrm{G}$, and $U_{\max }(15 \mathrm{~K}) \approx 50.8 \mathrm{meV}$ in $600 \mathrm{G}$. A percolation model predicts the formula $\mathrm{U}=\mathrm{B}+$ $\mathrm{AT}^{2}$ at $\mathrm{kT} \ll U$, and this observation on high- $\mathrm{T}_{\mathrm{C}}$ superconductors was reported by A. Gurevich et al. [8]. We observe a similar dependence of $U(T)$ in $T$ $\leq 11 \mathrm{~K}$. These results are similar to those reported by C.Pollitis et al. [8]. They observed that U(T) increases with $T$ when $T$ increases from $5 K$ to 20 $\mathrm{K}$, and $U$ can be well described by the formula $U=$ $\mathrm{B}+\mathrm{AT}^{2}$ with $\mathrm{B}$ and $\mathrm{A}$ independent of $\mathrm{T}$, while $\mathrm{U}$ we obtained increases with $\mathrm{T}^{2}$ linearly at $\mathrm{T} \leq 11 \mathrm{~K}$.

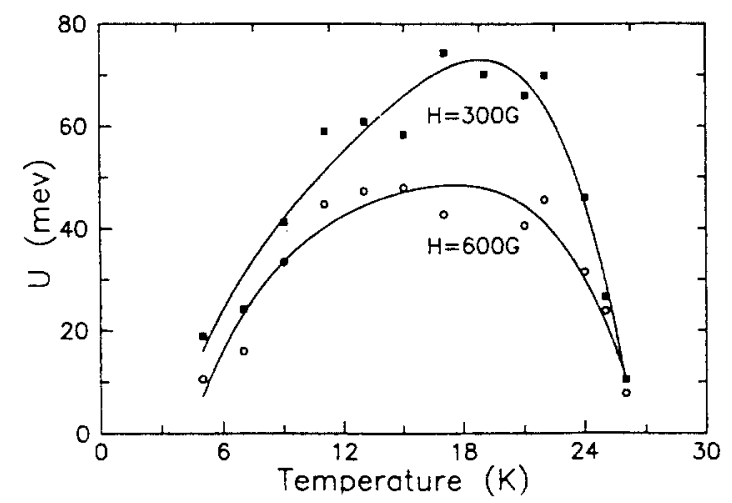

Figure2. Apparent pinning energy $U$ vs $T$ at 300 and $600 \mathrm{G}$.

To study the temperature dependence of the critical current density $\mathrm{J}_{\mathrm{C}}$. the following formula [10],

$$
\mathrm{J}_{\mathrm{C}} \propto(\mathrm{dM} / \mathrm{d} \ln \mathrm{t}) \times(\mathrm{U} / \mathrm{kT}),
$$

based on the Anderson-Kim model was used. Using $\mathrm{Eq}(2)$, we derive $\mathrm{J}_{\mathrm{C}}$ as a function of $\mathrm{T}$ as shown in fig[3], and Jc can be fitted to the formula, $J(T) \propto$ $\left(1-t^{n}\right)^{m}$, with $n \approx 3$ and $\mathrm{m} \approx 2.0$ in $600 \mathrm{G}$, where $\mathrm{t} \equiv$ T/Tc. Near $T_{c},\left(1-t^{n}\right) \approx n(1-t)$ for $t \rightarrow 1$, we thus expect the approximation $J_{C}(T) \propto[1-t]^{2}$. This result is analogous to the high-Tc superconductor $\mathrm{J}_{\mathrm{C}}(\mathrm{T}) \propto$ $[1-\mathrm{t}]^{\mathrm{p}}$ with $\mathrm{p}$ typically ranging from 1 to $5 / 2[10$ 11].

In summary we measure the magnetic relaxation of $\mathrm{C}_{60} \mathrm{Rb}_{3}$ in different temperature ranges in low magnetic fields. Using the flux creep model of Anderson-Kim, we derive the behavior of $U(T)$ and
$J_{\mathrm{C}}(T)$. The relaxation rate $S$ grows linearly with $\ln t$ for $t \geq 3600 \mathrm{sec}$. $U(T)$ show concave downward features. and $J_{C}(T) \propto(1-t)^{2}$ for $T$ near $T_{C}$.

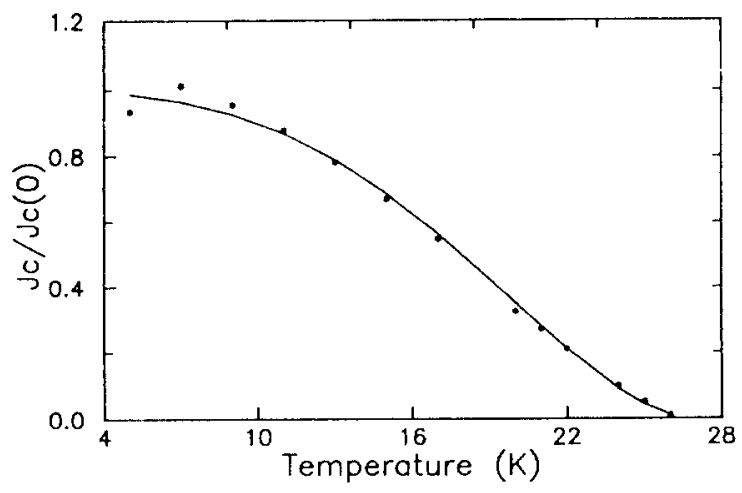

Figure3. The dependence of critical current density on temperature.

\section{REFERENCES}

1. K. Holczer, O. Klein, S. Huang, R.B. Kaner, K Fu. R.L. Whetten, and F. Diedench. Science 252, (1991) 11154 .

2. A.F. Hebard, M.J. Rosseinsky, R.C. Haddon, D.M. Murphy, S.H. Glarum, T.T. Mpalstra. A.P. Ramirez, and A.R. Kortan. Nuture 350 (1991) 600 .

3. M.J. Rosseinsky, A. P. Rcmivez, S.H. Glarum. D.W. Murphy, R.C. Haddon, A.F. Hebard. T.T.M. Palstra. A.R. Kurtan, S.M. Zahurak, and A.V. Makhijn, Phys. Rev. Lett. 66(1991)2830.

4. K. Holczer, O. Klein, G. Gruner, J.D. Thompson, F. Diederich and L. Whetten, Phys. Rev. Lett. 67 (1991) 271

5. V. Buntar, U. Eckern and C. Polities. Modern Phys. Lett. B. V6, N16 \& 17 (1992) 1037.

6. W. Kratschmer, L.D. Lamb, K. Fostiropoulos. and D. R.Huffman, Nature 347 (1990) 354.

7. P.W. Andersom, Phys. Rev. Lett. 9 (1962) 309 : Y.B. Kim, Rev. Mod. Phys. 36 (1964) 39.

8. C. Polits, V. Buntar, W. Krauss and A. Gurevich. Europhys. Lett. 17 (1992) 175.

9. A. Gurevich, H. Kupfer and C. Keller, Europhys Lett. 15 (1991) 789.

10. Y. Yeshurun and A.P. Malozemoff, Phys. Rev Lett. v60 n21 (1988) 2202.

11. M.V. Kartsornik et al., Pis'ma Zh. Eksp. Teor. Fiz. 47 ( I988) 595. [ JETP Lett. 47 (1988) 691]. 